\title{
Aplicación de tomografía de refracción sísmica y análisis de microtremores como técnicas de prospección geofísicas en estudios geotécnicos en edificación
}

\author{
Application of seismic refraction tomography \\ and microtremor analysis as geophysical prospecting \\ techniques in geotechnical studies in building
}

C. Pérez $^{(*)}$, M. Mateo ${ }^{(*)}$, A. Maciá ${ }^{(*)}$

\section{RESUMEN}

Aunque el CTE posibilita la aplicación de técnicas de prospección geofísicas como información complementaria a sondeos mecánicos para la caracterización geotécnica del terreno, su utilización en edificación está poco generalizada haciendo pertinente estudios que analicen las ventajas de su uso. Este artículo expone el análisis comparativo de los resultados obtenidos mediante una campaña experimental con técnicas de prospección geofísicas y tradicionales. La metodología empleada ha consistido en la caracterización de un terreno mediante tomografía de refracción sísmica con análisis de microtremores y sondeos mecánicos, comparándolos con los datos obtenidos durante la ejecución posterior de un recalce de cimentación en el mismo emplazamiento. Los resultados han permitido evaluar las limitaciones de cada método y ventajas de su combinación. En conclusión, la aplicación de técnicas de prospección geofísicas complementarias a sondeos mecánicos ha contribuido a una mejor caracterización geotécnica del terreno, mejorando la planificación y estimación de costes del proyecto de edificación.

480-7

Palabras clave: Técnicas de prospección geofísicas; tomografía de refracción sísmica; análisis de microtremores; estudio geotécnico.

\section{SUMMARY}

Although the CTE facilitates the application of geophysical prospecting techniques as complementary information to mechanical borings for the geotechnical characterization of the ground, its use in building is little widespread, making appropriate studies that analyze the benefits of its use. This article presents a comparative analysis of the results obtained using an experimental campaign with geophysical and traditional prospecting techniques. The methodo/ogy used has consisted in the characterization of a ground by seismic refraction tomography with microtremor analysis and mechanical borings, comparing them with the data obtained during the subsequent implementation of a foundation underpinning on the same site. The results have allowed to evaluate the limitations of each method and advantages of their combination.In conclusion, the application of geophysical prospecting techniques complementary to mechanical borings has contributed to a better geotechnical characterization of the ground, improving the planning and estimation of building project costs.

Keywords: Geophysical prospecting techniques; seismic refraction tomography; microtremor analysis; geotechnical study.

\footnotetext{
(*) Universidad de Alicante (España). 


\section{INTRODUCCIÓN}

La aplicación de técnicas de prospección geofísicas como la sísmica de refracción, permite la determinación de las características geotécnicas del terreno, siendo de gran utilidad tanto en ingeniería como en edificación. Su utilización queda recogida en las actuales normativas en España, como el Código Técnico de la Edificación (1). Sin embargo, su uso en nuestro país sigue siendo escaso en arquitectura y su validez queda limitada por la propia normativa sólo como información complementaria a los sondeos mecánicos.

La investigación aquí presentada surge de la necesidad de completar los resultados obtenidos mediante técnicas de prospección tradicionales para la realización de un proyecto de recalce estructural de la urbanización interior de un conjunto residencial de viviendas.

La urbanización se localiza próxima a la costa y, según un estudio geotécnico realizado mediante sondeos previamente al proyecto de reparación, se construyó sobre el cauce de un antiguo barranco con presencia de escorrentía subterránea que fue rellenado en épocas recientes con materiales altamente heterogéneos procedentes de excavaciones de la zona y vertidos de origen antrópico, como escombros y otros residuos de obras de construcción.

Las viviendas, que carecen de daños estructurales, fueron cimentadas a más de 7 metros de profundidad, empotradas en terreno firme constituido por materiales detríticos de elevada consistencia. Sin embargo, los pavimentos y muros de los espacios exteriores apoyan sobre el estrato superior de rellenos, y han sufrido graves desperfectos como consecuencia de los asientos diferenciales experimentados por la escasa compacidad de dicho suelo. De los datos obtenidos en el estudio geotécnico se deducía una estratigrafía del terreno aproximadamente horizontal que, sin embargo, no reflejaba la distribución irregular de los daños detectados en las diferentes zonas de la parcela.

Por ello, y considerando que la gran superficie de terreno a estudiar hacía económicamente inviable plantear la realización de más sondeos para la obtención de datos más precisos, se decidió recurrir a técnicas de prospección geofísica, combinando al menos dos métodos tal y como defienden algunos autores (2), para determinar si la distribución real de los distintos niveles estratigráficos en toda la superficie de la parcela era más irregular que la reflejada en el estudio geotécnico tradicional de sondeos, y así poder prever con mayor exacti- tud la longitud final total de los micropilotes a ejecutar para el recalce estructural. Los métodos seleccionados han sido la tomografía sísmica de refracción por un lado y la escucha pasiva de microtremores por otro.

Mediante la técnica de la tomografía, desarrollada en Sismología de forma simultánea al campo de la Medicina desde 1976 (3) y que ya obtuvo los primeros resultados utilizando métodos iterativos en los primeros años ochenta (4), se obtienen imágenes de secciones planas del interior del terreno. El método de refracción sísmica empleado resulta adecuado para el tipo de investigación realizada, cuyo objeto es localizar la cota de profundidad de la capa de roca resistente situada bajo un recubrimiento superficial de terreno alterado y meteorizado, originándose un contraste en la velocidad sísmica (5).

Por su parte, el análisis de microtremores se trata de una técnica muy empleada recientemente en geofísica y avalada por múltiples autores (6) como un método más barato y rápido para la obtención del perfil estratigráfico en materiales superficiales y rocas subyacentes (7) (8) (9) (10), que constituye una alternativa técnica para determinar la velocidad de propagación $\mathrm{V}_{\mathrm{s}}$ de las ondas $\mathrm{S}$ mediante los ensayos down-hole y cross-hole (11) recomendados por la norma DB-SE-C del CTE.

El empleo de técnicas de prospección geofísica en edificación y en el medio urbano obliga a la adaptación de los dispositivos y equipamiento de toma de datos a espacios de intervención bastante reducidos que dificultan el empleo de determinadas técnicas (12), debiéndose considerar además la afección que sobre el rendimiento de los métodos empleados pueden tener los campos físicos de origen artificial como vibraciones o corrientes eléctricas, y las dificultades que sobre las metodologías a emplear supone la existencia de firmes o pavimentos superficiales.

El estudio comparativo realizado de las diferentes técnicas empleadas y el análisis de sus resultados supone una contribución a la comprensión de las posibilidades que puede tener la acción combinada de las diferentes técnicas de prospección geofísica y tradicionales, en aras a favorecer su difusión y aplicación generalizada en el sector de la edificación.

\section{MATERIAL Y MÉTODOS}

\subsection{Metodología}

La metodología empleada se ha basado en la aplicación, en un mismo terreno soporte, 
de técnicas de prospección geofísica y técnicas de prospección tradicionales, y en el análisis comparativo de los resultados obtenidos con los datos reales de las características del terreno emanados de la ejecución posterior de un proyecto de reparación de la urbanización del conjunto residencial consistente en la consolidación del suelo y recalce estructural con cimentación profunda mediante micropilotaje realizada en el mismo emplazamiento.

Las técnicas de prospección geofísica empleadas han consistido en la realización de una tomografía de refracción sísmica con la ejecución de 5 perfiles en 2D obteniendo la distribución vertical de la velocidad de ondas primarias $V_{p}$ y adicionalmente la realización de 4 registros de microtremores para aportar información complementaria acerca de la evolución media de la velocidad de ondas de cizalla $V_{s}$.

El interés de la investigación se ha centrado en la estimación de la distribución vertical de la velocidad de las ondas longitudinales (P) a lo largo de varios perfiles con la intención de detectar posibles anomalías o discontinuidades laterales en el terreno. Adicionalmente se trataba de valorar la evolución vertical media de la velocidad de ondas de cizalla con vistas a la clasificación geotécnica del lugar.

El método de tomografía de refracción sísmica empleado combina tendidos de refracción convencional de facilidad de medida y despliegue, y técnicas de inversión de los tiempos de viaje de la señal sísmica denominadas Wet Inversion y Wet Tomography (Wavepath Eikonal Tomography) (13), que proporcionan imágenes bidimensionales de la distribución de las velocidades de las ondas primarias en el subsuelo mediante métodos informáticos basados en estimaciones numéricas de modelos de velocidad óptima de los datos medidos.

La técnica de análisis de microtremores empleada para la obtención de los perfiles verticales de la velocidad de ondas de cizalla se basa en el análisis del ruido sísmico estacionario provocado por una serie de acciones artificiales capaces de producir pequeñas vibraciones en el terreno, permitiendo analizar el comportamiento del suelo frente a esfuerzos verticales y de cizalla.

Los modos de vibración resultantes están condicionados por las características mecánicas de las capas de subsuelo comprendidas entre la superficie y los primeros 50 a 100 metros de profundidad. La metodología empleada combina los datos obtenidos mediante perfiles de refracción clásicos y su transformación matemática capaz de ofrecer una buena visualización de la curva de dispersión de los modos principales de las ondas superficiales $\left(R_{g}\right)$.

Mediante la inversión de la curva de dispersión de las ondas superficiales se obtiene un modelo vertical de velocidades de ondas de cizalla que, además de su interés geotécnico de cara al cálculo del coeficiente sísmico del terreno conforme la norma NCSE/02 (14), permite representar los valores promedio de este parámetro en la sección comprendida por el perfil, por lo que proporciona una estimación inicial del valor medio de la profundidad de las diferentes interfaces o superficies de fuerte gradiente de velocidad, cuya posición detallada debajo de cada geófono o punto de medida es estimada en la tomografía.

Las técnicas de prospección tradicionales empleadas han consistido en el reconocimiento del subsuelo mediante la realización de tres sondeos a rotación con recuperación continua de testigo, distribuidos en la parcela (Figura 1). Se han perforado un total de 40,22 metros lineales distribuidos de la siguiente manera: 9,85 m. en el sondeo 1, 15,25 m. en el sondeo 2 y 15,12 m. en el sondeo 3 .

Durante los trabajos de perforación se han realizado también un total de 19 Ensayos Normalizados de Penetración Dinámica (S.P.T.) conforme norma UNE-EN ISO 22476-3:2006 (15), y se han obtenido 4 muestras de carácter inalterado de aqueIlos materiales de características limosas mediante la hinca de Toma-muestras Normalizados de pared gruesa según norma ASTM D1587-08 (16).

Los ensayos en laboratorio de los testigos extraídos en los sondeos han incluido análisis granulométrico por tamizado y sedimentación, y determinación de plasticidad, contenido de humedad natural, peso específico, densidad aparente, colapsabilidad potencial y contenido de sulfatos.

El proyecto de reparación de la urbanización ha consistido en la consolidación del suelo y recalce estructural de las zonas pavimentadas de la urbanización, quedando excluidos los edificios, mediante la realización de cimentación profunda consistente en micropilotes de hormigón armado y posterior ejecución de losas continuas de hormigón armado como estructura horizontal de soporte de los pavimentos a reconstruir.

Los micropilotes están distanciados uniformemente entre ellos formando una retícula 
1. Distribución de los perfiles en la parcela.

2. Disposición de los geófonos.

3. Conexión geófonos para registro de datos.

4. Registro de datos de los geófonos.
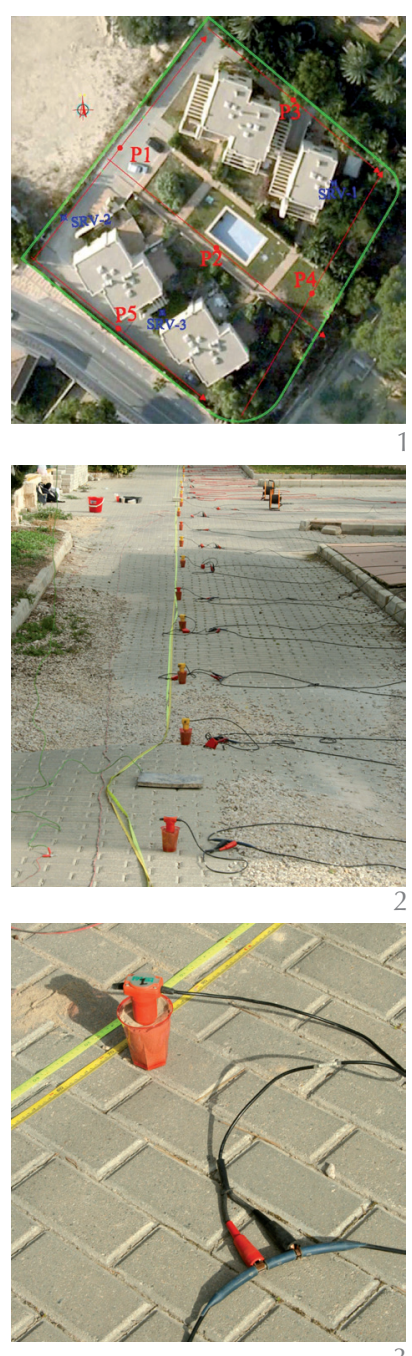

En cada perfil se han dispuesto 24 geófonos espaciados 2,5 metros en el primer caso y 2,0 metros en el segundo (Figura 2).

Para el registro de los datos, los geófonos estaban conectados a través de un cable de señales a un registrador digital Seistronic RAS24 (Figura 3), de ganancia automática y preamplificación de $24 \mathrm{~dB}$, con longitud de registro 0,20 mseg., muestreo de 0,25 mseg. y 24 canales, controlado desde un PC portátil, lo que permite mediciones con entendimientos de más de tres tiros y facilita

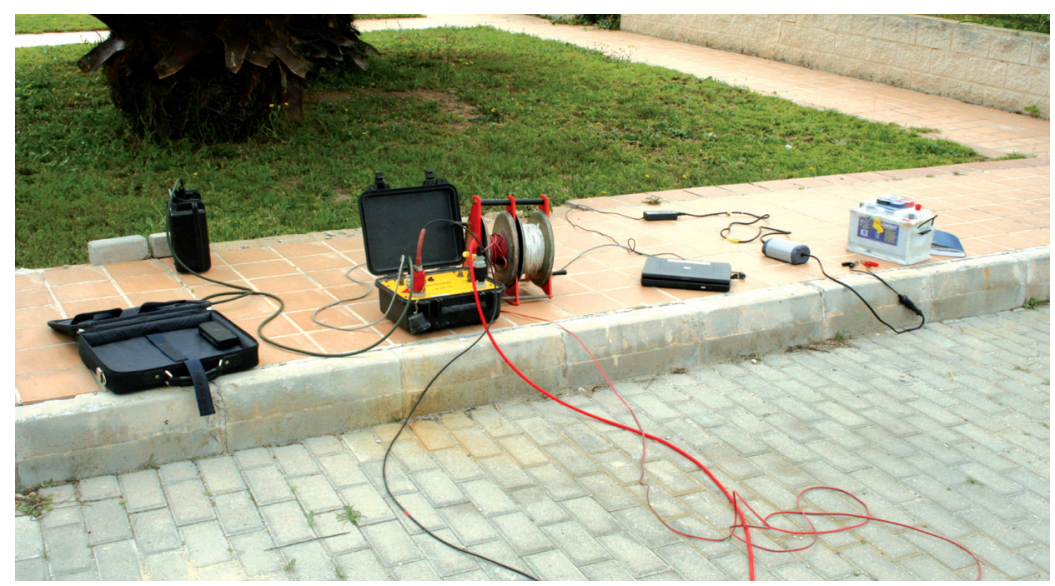

al operador intuir las domocrónicas almacenando los registros de campo para una determinación más precisa y correcta de los tiempos de las primeras Ilegadas mediante la posterior utilización de programas informáticos que permiten filtrar e individualizar los registros obtenidos (5) (Figura 4).

Cada tiro se ha repetido 2 veces para mejorar la relación señal/ruido. La prueba ha sido realizada por el Área de Física de la Tierra del Departamento de Ciencias de la Tierra de la Universidad de Alicante.

Para la realización del análisis de microtremores mediante el registro in situ de los datos se ha utilizado una de las líneas o tendido de la tomografía de refracción sísmica realizada (perfil P4) atribuyéndose los resultados al punto medio del perfil, tomando 4 registros de ruido sísmico de 32 segundos de duración, con diferentes preamplificaciones que combinados han dado lugar al registro de microtremores o ruido sísmico $\mathrm{R} 4$.

\section{RESULTADOS}

Las técnicas de prospección tradicionales mediante sondeos han permitido detectar y clasificar los distintos niveles del terreno, definiendo con precisión los parámetros y características geotécnicas de cada uno de ellos.

El primer nivel (Nivel I), que comprende desde la superficie hasta una profundidad aproximada comprendida entre los 5,80 y los 6,20 m según la zona, se caracteriza por la presencia de materiales altamente heterogéneos con sedimentos procedentes de la excavación de materiales naturales de la zona (gravas, limos, arcillas, etc.) junto con otros de clara influencia antrópica (restos de ladrillo, cascotes, hormigón visto, vidrio, metal, etc.). La calidad de estos materiales resulta pésima como terreno soporte para edificación debido a su alta heterogeneidad, su escasa compacidad evidenciada con valores de golpeo en los ensayos S.P.T. entre 4 y 8 golpes, y su alto contenido de humedad.

El segundo nivel detectado (Nivel II), consistente en materiales detríticos gruesos como arenas y gravas así como con un importante contenido en limos, sí presenta una elevada resistencia a la penetración, como se desprende de los resultados de los ensayos de penetración estándar efectuados en los sondeos mecánicos (S.P.T.). Este segundo nivel se sitúa desde los 5,80-6,20 metros de profundidad hasta los 9,30-13,25 metros de profundidad según la localización del sondeo. 
El tercer nivel (Nivel III) localizado por debajo del nivel anterior, consistente en limos arenosos y arenas limosas, presenta igualmente una alta consistencia.

Los resultados quedan reflejados en las columnas litoestratigráficas del estudio geotécnico (Figura 5), así como en los perfiles litológicos realizados a partir de los datos de los sondeos, obteniéndose los perfiles 1-2 y 2-3 (Figura 6).

Las técnicas de prospección geofísica mediante tomografía de refracción sísmica han permitido identificar los distintos niveles del terreno en función de la relación entre la velocidad de propagación de las ondas y las características geotécnicas de cada uno de ellos. La representación gráfica de los datos obtenidos permite visualizar, a lo largo de los cinco perfiles realizados, la distribución en profundidad de los estratos con igual velocidad de transmisión. Las gráficas representan en escala de colores la velocidad de transmisión, referenciada respecto a la distribución de geófonos representados en el eje $X$, y la profundidad de los estratos representada en el eje $Y$ (Figuras 7, 8, 9, 10 y 11).

Adicionalmente, el método de microtremores aplicado en el perfil P4, ha permitido asignar valores teóricos a la velocidad de ondas de cizalla. A partir de la interpretación y procesado de los datos de campo, se
5. Columnas litoestratigráficas del estudio geotécnico correspondientes a los sondeos 1, 2 y 3.

6. Perfiles litológicos del estudio geotécnico correspondiente a los sondeos $1-2$ y $2-3$.
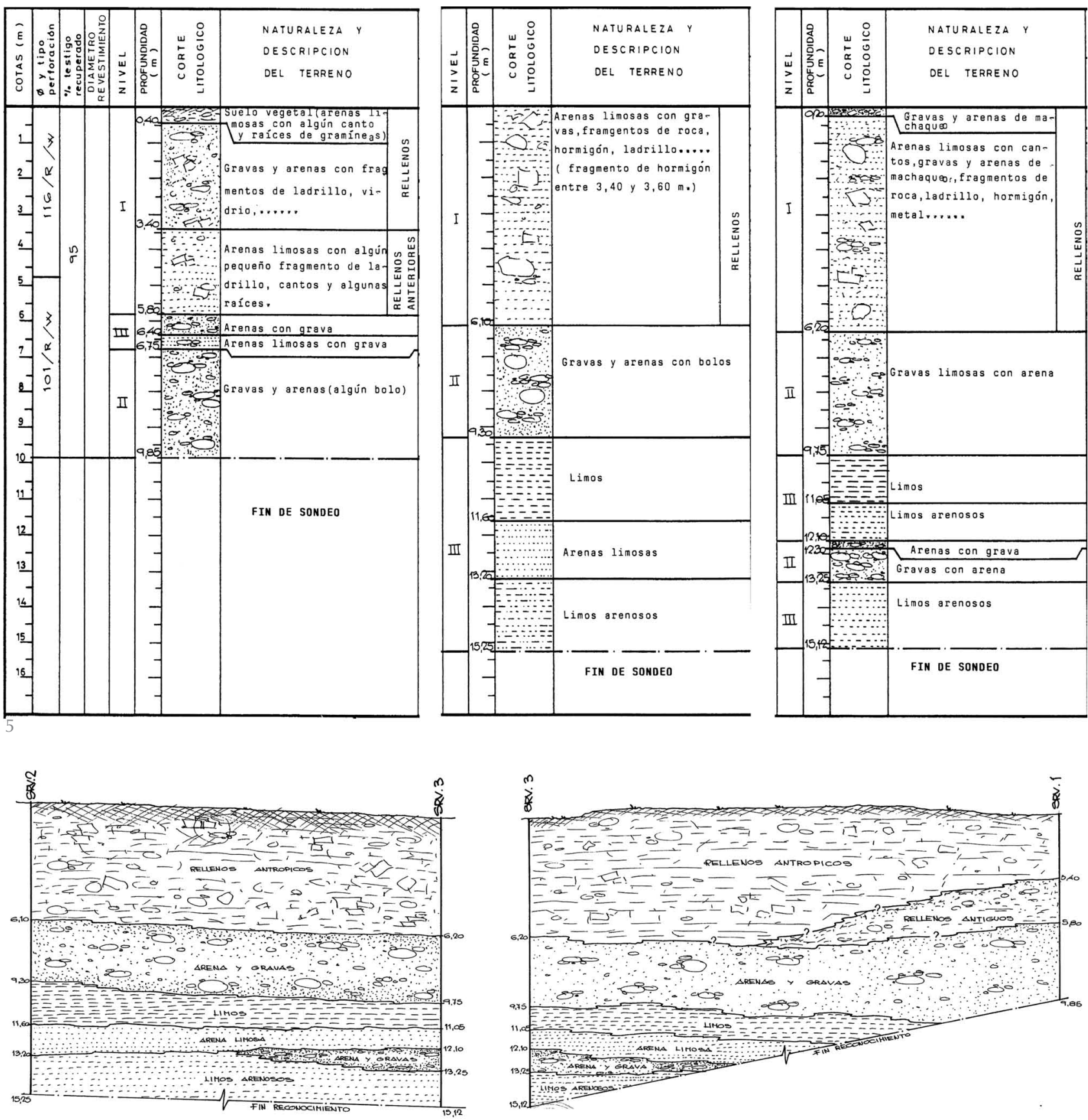

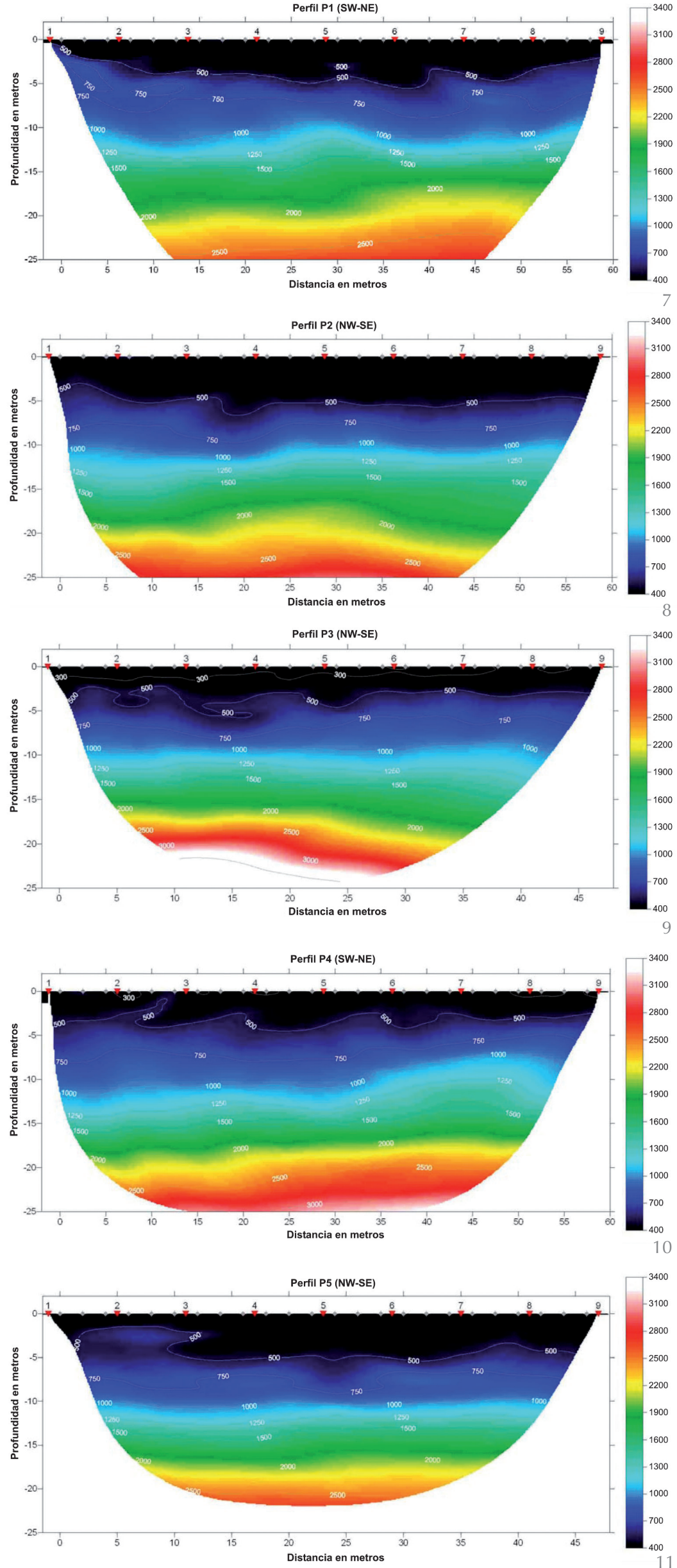

ha procedido a la transformación del registro distancia/tiempo (dominio $x / t$ ) a lentitud/frecuencia (dominio $\mathrm{p} / \mathrm{f}$ ), con el objeto de resaltar eventos de naturaleza dispersiva como las ondas de superficie y estimar la tendencia del modo principal de dispersión de ondas Rayleigh (Figura 12). Con la inversión de la curva de dispersión obtenida mediante ajuste interactivo a las curvas de dispersión teóricas y la resolución de la curva en capas de diferente velocidad (Figura 13), se ha procedido a representar el modelo vertical de velocidad de ondas de cizalla (Figura 14).

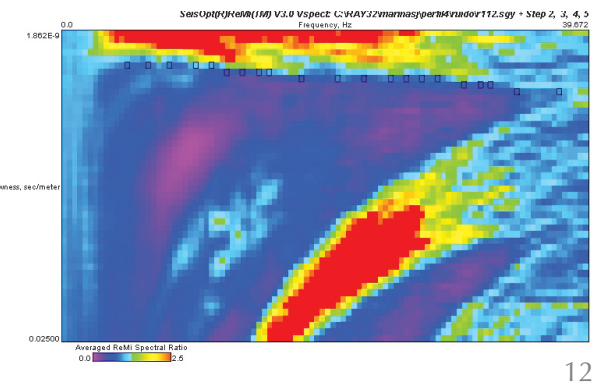

El análisis conjunto de todos estos resultados junto con las columnas litológicas obtenidas en el estudio geotécnico mediante sondeos, ha permitido realizar una interpretación geofísica de los datos obtenidos procediendo a la atribución litológica y estimación de parámetros de los diferentes estratos identificados.

La comparación entre la distribución de velocidades de transmisión Vp y Vs detectadas y los estratos de terreno identificados y clasificados en el estudio geotécnico, permite correlacionar los distintos datos obtenidos identificando tres unidades sismo-estratigráficas. La primera unidad estratigráfica, en los primeros 5-7 metros de profundidad, está caracterizada por velocidades $V p$ desde 300 hasta $600 \mathrm{~m} / \mathrm{s}$ y velocidades Vs desde 200 hasta $300 \mathrm{~m} / \mathrm{s}$, y corresponde a la capa de rellenos antrópicos. La segunda unidad estratigráfica, comprendida desde los 5-7 hasta los 10-12 metros de profundidad, está caracterizada por velocidades $\mathrm{Vp}$ de $800-1000 \mathrm{~m} / \mathrm{s}$ y velocidades Vs de $450-550 \mathrm{~m} / \mathrm{s}$, y corresponde a la capa de gravas y arenas. La tercera unidad estratigráfica, comprendida desde los 10-12 hasta los 20-25 metros de profundidad, está caracterizada por velocidades $V p$ de $1000-1500 \mathrm{~m} / \mathrm{s}$ y velocidades $V$ s de $400-500 \mathrm{~m} / \mathrm{s}$, y corresponde a la capa de limos y arenas consolidados.

Los resultados obtenidos de las técnicas de prospección geofísica empleadas manifiestan una mayor irregularidad de la superficie de contacto entre la capa de rellenos y la de gravas que en el resto de estratos, caracterizados por una estratificación más horizontal. Dicha irregularidad evidencia el lavado de finos de 
PerfilR4

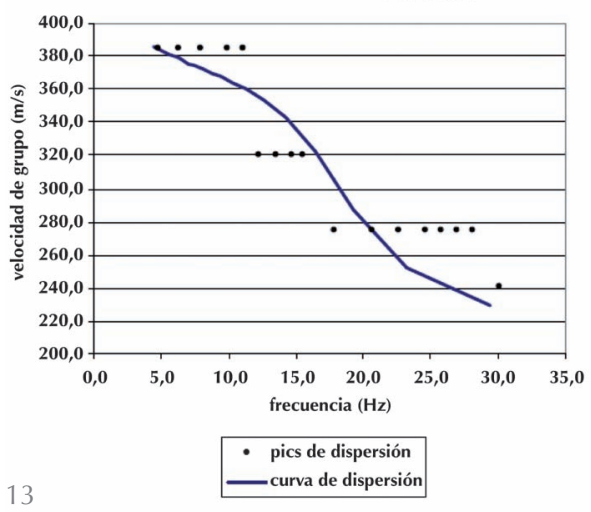

la base de los rellenos a través de la capa inferior de gravas debido a su elevada permeabilidad, lo que explica los asientos por gravedad detectados en la capa de rellenos.

Finalmente, la ejecución de los micropilotes durante la obra de recalce estructural del conjunto residencial evidencia importantes irregularidades en la profundidad de la zona de transición entre la capa de rellenos antrópicos y la capa de gravas y arenas considerada como estrato competente para la transmisión de cargas mediante cimentación profunda.

La distribución y longitudes de los micropilotes, realizados hasta su empotramiento en estrato considerado firme, muestra una profundidad media de la superficie superior de la capa de gravas y arenas comprendida entre los 7 y los 12 metros, lo que arroja resultados concordantes a los datos obtenidos de los sondeos y la tomografía, pero hace manifiesta la existencia de zonas puntuales con hundimientos próximos a los 25 metros de profundidad (Figura 15).

\section{DISCUSIÓN}

A partir de los cinco perfiles litológicos obtenidos (P1, P2, P3, P4 y P5), se ha procedido a representar gráficamente las diferentes profundidades estimadas y reales de la capa de gravas y arenas, unidad estratigráfica Nivel II considerada competente para el apoyo y transmisión de cargas de la cimentación (Figuras 16, 17, 18, 19 y 20).

Por una parte, se muestran las profundidades estimadas en los perfiles simplificados propuestos por el estudio geotécnico, deducidos con elevado margen de error a partir de los datos obtenidos en los tres sondeos realizados. Por otra parte, se ven las profundidades obtenidas de la tomografía de refracción sísmica considerando la isolínea de $800 \mathrm{~m} / \mathrm{s}$ como la velocidad $V p$ correspondiente a las gravas. $Y$ finalmente, se representan las verdaderas profundidades de empotramiento en estrato firme obtenidas durante la realización de los micropilotes.

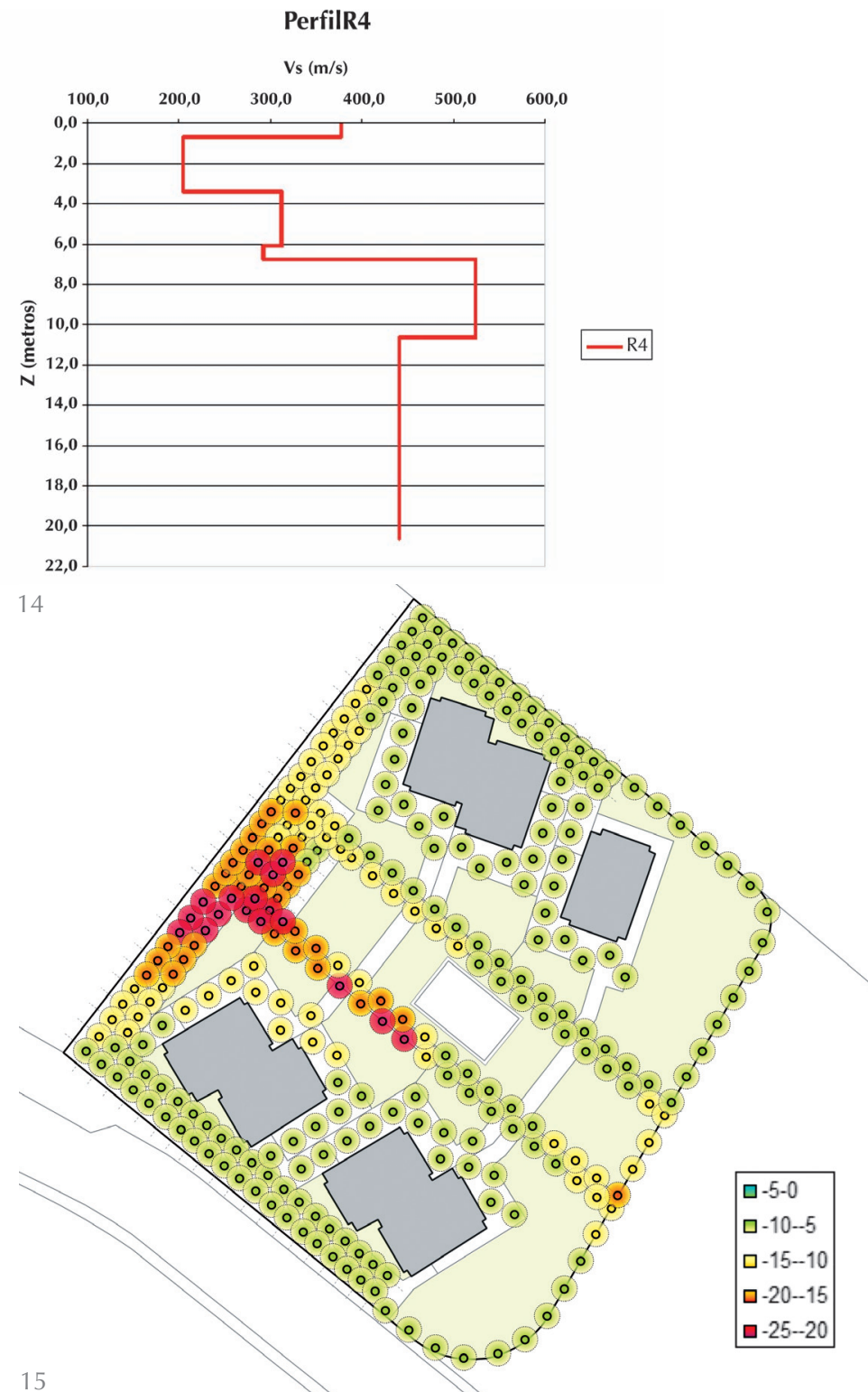

El análisis comparativo de los resultados obtenidos permite constatar los desiguales niveles de precisión y limitaciones de los diferentes métodos de prospección empleados en este estudio.

Partiendo de los resultados obtenidos y, mediante interpolación de datos, se ha procedido a modelizar tridimensionalmente las profundidades del estrato considerado firme, según las previsiones a partir de los datos obtenidos de los sondeos y la tomografía, y según los datos reales obtenidos tras la ejecución de los micropilotes (Figura 21).

Como se aprecia en la comparativa entre los resultados obtenidos con las diferentes técnicas, los sondeos mecánicos han permitido obtener datos precisos y veraces sobre las características geotécnicas de los estratos y sus profundidades, pero sólo en los puntos de realización de los sondeos. La validez y exactitud de estos datos queda demostrada por su elevado grado de coin-
7. Tomografía correspondiente al perfil $\mathrm{P} 1$.

8. Tomografía correspondiente al perfil P2.

9. Tomografía correspondiente al perfil P3.

10. Tomografía correspondiente al perfil P4.

11. Tomografía correspondiente al perfil P5.

12. Transformada $\mathrm{p} / \mathrm{f}$ del registro R4 (perfil P4) y curva de dispersión estimada.

13. Ajuste entre curvas de dispersión empírica y teórica R4 (perfil P4).

14. Modelo vertical de velocidad de ondas de cizalla R4 (perfil P4).

15. Distribución y profundidad de empotramiento de cada micropilote en la unidad geotécnica resistente. 

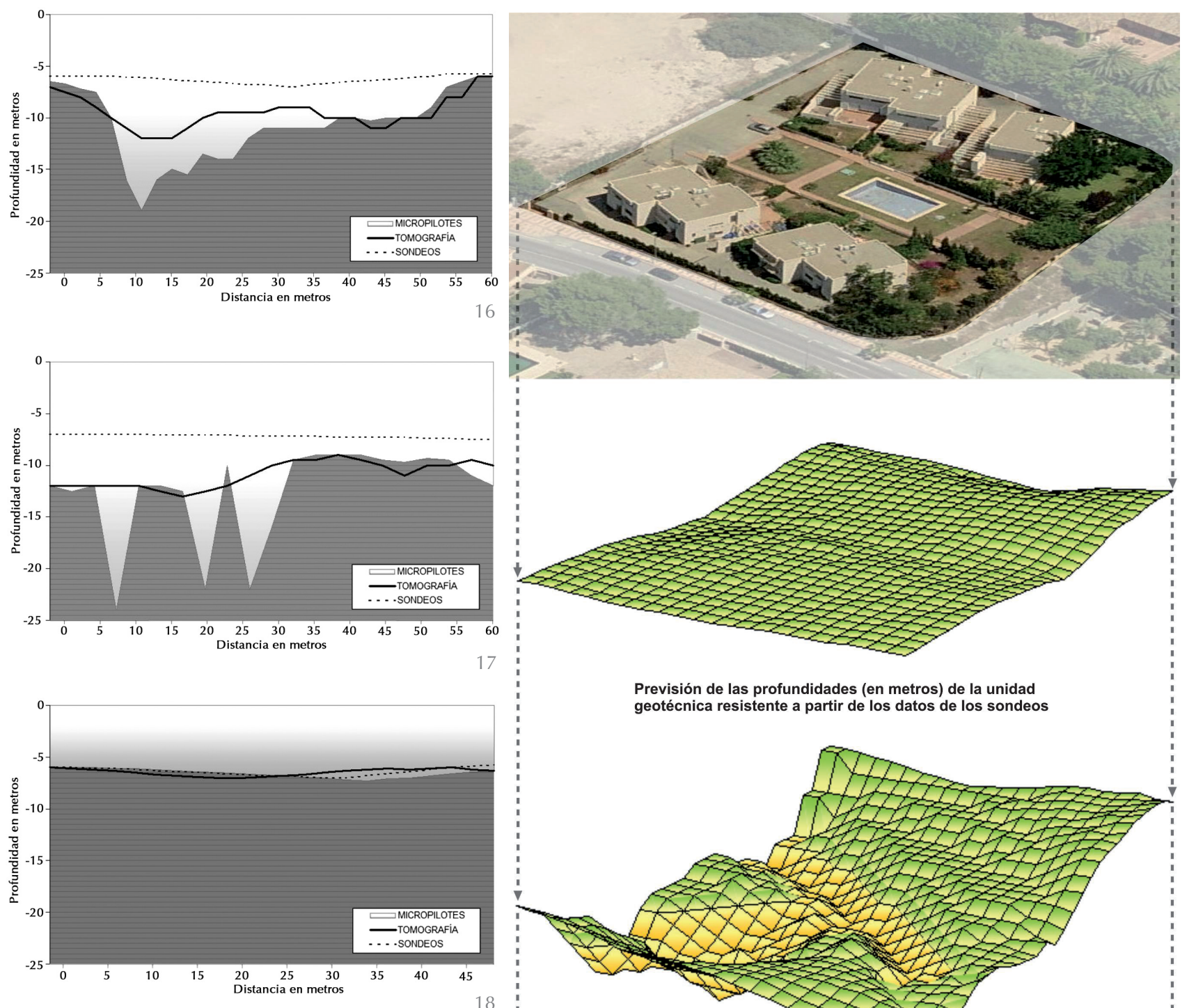

Previsión de las profundidades (en metros) de la unidad geotécnica resistente a partir de los datos de los sondeos
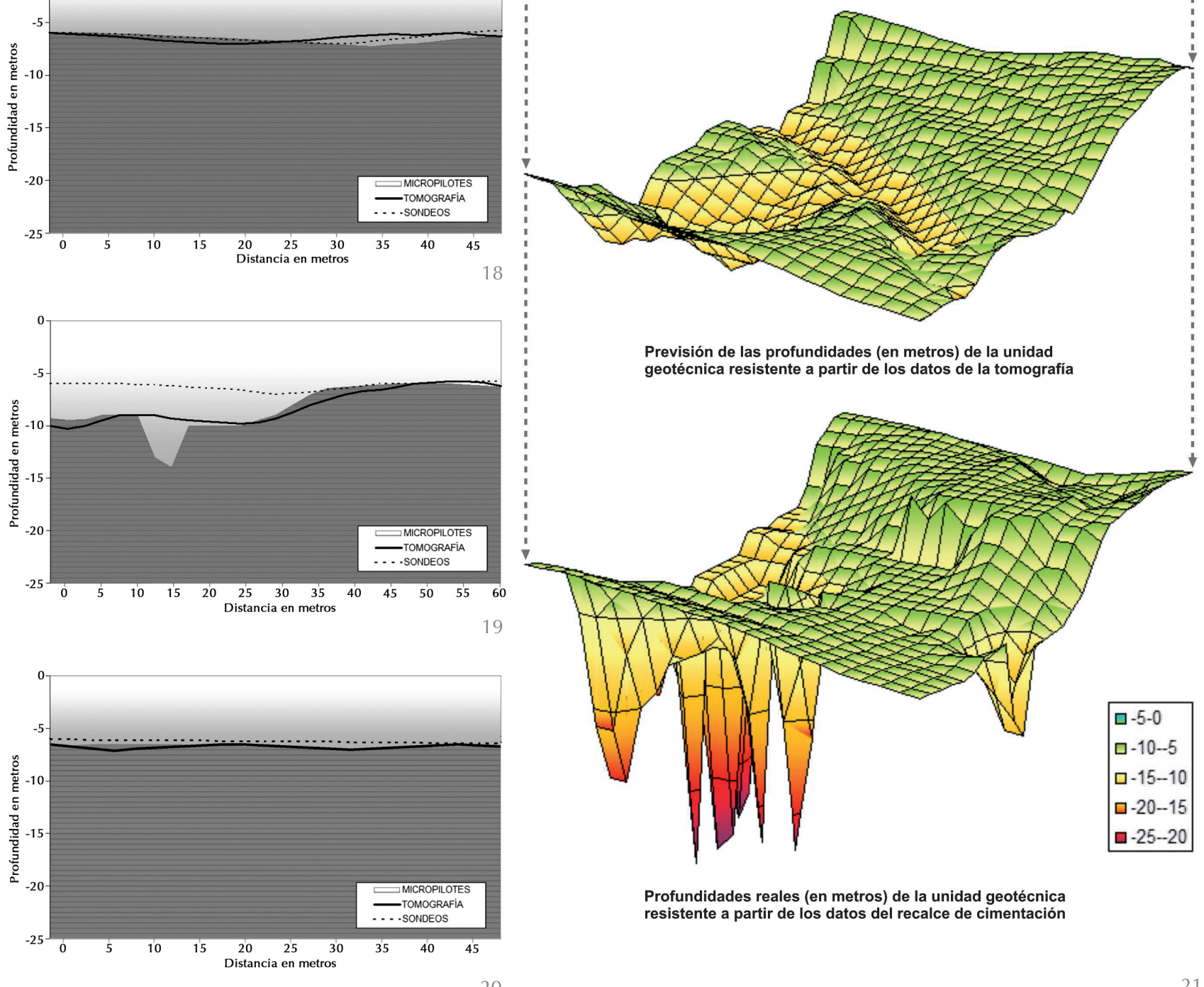

Profundidades reales (en metros) de la unidad geotécnica resistente a partir de los datos del recalce de cimentación 
cidencia con los datos reales obtenidos durante la ejecución posterior de los micropilotes de la obra de recalce de cimentación. Sin embargo, la no viabilidad material ni económica de realizar gran cantidad de sondeos, debido al coste de los medios materiales y humanos que requiere este tipo de técnica, impide detectar la existencia de notables anomalías geotécnicas en otras zonas de la parcela. Como se ha podido comprobar en el caso de estudio, los tres sondeos realizados, distribuidos estratégicamente de manera equidistante y amoldándose a la distribución de las edificaciones, no han permitido detectar las graves alteraciones del terreno soporte existentes en la zona central de la parcela resultando erróneas las estimaciones de los perfiles litológicos deducidos a partir de los sondeos realizados en el estudio geotécnico.

Por su parte, los resultados de la tomografía de refracción sísmica y el análisis de microtremores han permitido detectar cambios importantes en la profundidad de la unidad geotécnica resistente cuando éstos afectan a una superficie de terreno considerablemente representativa. Sin embargo, estas técnicas geofísicas tampoco han permitido detectar cambios de profundidad en los estratos litológicos cuando éstos se producen de manera muy localizada y con buzamientos importantes, corroborando las limitaciones de aplicación que el CTE establece para estas técnicas al prescribirlas sólo para formaciones estratigráficas relativamente horizontales (buzamiento inferior a $15^{\circ}$ ). En el caso de estudio, los métodos de sísmica de refracción empleados no detectaron las importantes afecciones puntuales en el subsuelo producidas en los estratos profundos del terreno, generadas como consecuencia del lavado de finos producido en la base de la capa de rellenos a través de la capa de gravas causante de los importantes asientos experimentados por los rellenos antrópicos del Nivel I, tal y como posteriormente sí quedó confirmado en el incremento notable de las profundidades alcanzadas puntualmente por algunos micropilotes hasta empotrar en el estrato resistente.

En general, se constata cómo los resultados de los sondeos y la tomografía de refracción sísmica sí se han aproximado mucho a los datos reales en las zonas de terreno que presentan estratos más homogéneos y de profundidad aproximadamente constante.

El análisis comparativo del conjunto de resultados obtenidos permite verificar cómo, pese a la mayor precisión de los datos geotécnicos obtenidos en los son- deos, la previsión de las profundidades de la unidad geotécnica resistente a partir de las estimaciones simplificadas de los perfiles litológicos del estudio geotécnico, permite calcular una desviación en torno al $28,94 \%$ respecto de las profundidades reales obtenidas a partir de los datos de la obra de recalce de cimentación.

Por su parte, a pesar de la menor precisión de los datos geotécnicos obtenidos mediante la tomografía de refracción sísmica y análisis de microtremores, la previsión de las profundidades de la unidad geotécnica resistente a partir de los datos de las técnicas geofísicas empleadas, permite reducir la desviación en torno al 3,84\% respecto de las profundidades reales obtenidas del recalce estructural, concentrándose dicha desviación en las zonas puntuales del terreno con graves afecciones en el subsuelo no detectadas por los métodos de sísmica de refracción.

La discusión de los resultados permite corroborar la contribución de la aplicación de las técnicas de prospección geofísicas empleadas para la obtención de información complementaria adecuada para la correcta interpretación de los datos geotécnicos obtenidos mediante los sondeos mecánicos, reduciendo notablemente el margen de error en la estimación de las profundidades de la unidad geotécnica resistente derivadas de los perfiles litológicos deducidos a partir de los sondeos realizados en el estudio geotécnico, y habiendo posibilitado una mejor planificación del recalce de cimentación y estimación de costes más ajustada del proyecto de edificación realizado.

La evaluación de las limitaciones de los diferentes métodos de prospección empleados muestra cómo la aplicación combinada de técnicas geofísicas y sondeos mecánicos permite a su vez una mejor distribución de los puntos de reconocimiento y reducir el margen de error derivado de cada técnica, especialmente en terrenos poco homogéneos y estudios geotécnicos de grandes superficies a edificar susceptibles de tener diferentes profundidades de la unidad geotécnica resistente.

\section{CONCLUSIÓN}

El análisis y discusión de los resultados obtenidos permite comprobar cómo la aplicación de técnicas de prospección geofísicas de forma complementaria a sondeos mecánicos ha contribuido a una mejor caracterización geotécnica del terreno, corrigiendo las estimaciones simplificadas y erróneas de los perfiles litológicos dedu-
16. Comparativa correspondiente al perfil P1 (SW-NE).

17. Comparativa correspondiente al perfil P2 (NW-SE).

18. Comparativa correspondiente al perfil P3 (NW-SE)

19. Comparativa correspondiente al perfil P4 (SW-NE).

20. Comparativa correspondiente al perfil P5 (NW-SE).

21. Comparativa entre la previsión de las profundidades de la unidad geotécnica resistente según los resultados obtenidos mediante sondeos y tomografía, y los datos reales obtenidos del recalce de cimentación. 
cidos a partir de los sondeos realizados, y posibilitando una mejor planificación del recalce de cimentación y estimación de costes más ajustada del proyecto de edificación ejecutado.

En conclusión, el estudio realizado ha permitido evaluar las limitaciones de los diferentes métodos de prospección empleados, y corroborar las ventajas de la aplicación combinada de técnicas geofísicas como la tomografía de refracción sísmica y el análisis de microtremores junto con técnicas de prospección tradicionales como los sondeos mecánicos, para la obtención de información complementaria óptima para la correcta interpretación de los datos geotécnicos obtenidos, contribuyendo al perfeccionamiento metodológico de las técnicas de reconocimiento del terreno habitualmente empleadas en los estudios geotécnicos en edificación.

\section{REFERENCIAS}

(1) Documento Básico DB-SE-C Seguridad Estructural Cimientos, Código Técnico de la Edificación. pp. 13 y 110-111, Ministerio de la Vivienda, Gobierno de España, 2006.

(2) Schrott, L., Sass, O. (2008). Application of field geophysics in geomorphology: Advances and limitations exemplified by case Studies. Geomorphology, 93(1-2): 55-73. doi: 10.1016/j.geomorph.2006.12.024.

(3) Aki, K., Christofferson, A., Husebye, E.S. (1977). Determination of the three dimensional seismic structure of the lithosphere. Journal of Geophysical.Research, 82(2): 277296. doi: 10.1029/JB082i002p00277.

(4) Clayton, R.W., Comer, R.P. (1983). A tomographic analysis of mantle heterogeneities from body wave travel times. EOS, Transactions, American Geophysical Union, 62:776.

(5) Orellana, E., Higueras, M., Merchán, F. (1995). La interpretación automática (por medio de ordenador) en la prospección geofísica. Casos de refracción sísmica y de SEV. Física de la Tierra, 7: 33-51.

(6) Louie J. (2001). Faster, better: shear-wave velocity to 100 meters depth from refraction microtremor arrays. Bulletin of the Seismological Society of America, 91(2): 347-364, doi: 10.1785/0120000098.

(7) Coccia, S., del Gaudio, V., Venisti, N., Wasowski, J. (2010). Application of Refraction Microtremor (ReMi) technique for determination of 1-D shear wave velocity in a landslide area. Journal of Applied Ceophysics, 71(2-3): 71-89, doi: 10.1016/j. jappgeo.2010.05.001.

(8) Pancha, A., Anderson, J.G., Louie, J.N., Pullammanappallil, S.K. (2008). Measurement of shallow shear wave velocities at a rock site using the ReMi technique. Soil Dynamics and Earthquake Engineering, 28(7): 522-535, doi:10.1016/j.soildyn.2007.08.005.

(9) Socco, L., Foti, S., Boiero, D. (2010). Surface-wave analysis for building near-surface velocity models - Established approaches and new perspectives. Geophysics, 75(5): 75A83-75A102, doi:10.1190/1.3479491.

(10) Raines, M.G., Gunn, D.A., Morgan, D.J.R., Williams, G., Williams, J.D.O., Caunt, S. (2011). Refraction microtremor (ReMi) to determine the shear-wave velocity structure of the near surface and its application to aid detection of a backfilled mineshaft. Quarterly Journal of Engineering Geology and Hydrogeology, 44(2): 211-220, doi: 10.1144/14709236/09-046.

(11) ASTM D4428 / D4428M-07, Standard Test Methods for Crosshole Seismic Testing, American Society for Testing and Material, EEUU, 2007.

(12) Moreno, J. (2010). Patologías en edificación: nuevas tecnologías geotécnicas y geofísicas para su auscultación. Tierra y tecnología, 37: 63-73.

(13) Schuster, G.T., Quintus-Bosz, A. (1993). Wavepath eikonal traveltime inversion: Theory. Geophysics, 58(9): 1314-1323, doi:10.1190/1.1443514.

(14) REAL DECRETO 997/2002, de 27 de septiembre, por el que se aprueba la norma de construcción sismorresistente: parte general y edificación (NCSR-02). Ministerio de Fomento, Gobierno de España, 2002.

(15) UNE-EN ISO 22476-3:2006, Investigación y ensayos geotécnicos. Ensayos de campo. Parte 3: Ensayo de penetración estándar (ISO 22476-3:2005), Asociación Española de Normalización y Certificación AENOR, España, 2006.

(16) ASTM D1587-08, Standard Practice for Thin-Walled Tube Sampling of Soils for Geotechnical Purposes, American Society for Testing and Material, EEUU, 2008. 